\title{
The hypercentral Constituent Quark Model
}

\author{
M.M. Giannini \\ Dipartimento di Fisica dell'Università di Genova \\ and \\ I.N.F.N., Sezione di Genova \\ E. Santopinto \\ I.N.F.N., Sezione di Genova
}

\begin{abstract}
The hypercentral Constituent Quark Model is presented and its application to the description of the electromagnetic properties of baryons is reviewed. The results concerning the elastic nucleon form factors and the electromagnetic excitation of baryon resonances are compared with the recent experimental data
\end{abstract}

\section{Introduction}

The hypercentral Constituent Quark Model (CQM) has been proposed some years ago [1] and it has been applied to the description of non strange baryons. The hCQM contains, as it will be seen, only three free parameters, which are fitted to the baryon spectrum. Once the parameters have been fixed, the model is completely determined and its results provide predictions for the baryons properties. In particular we shall consider the helicity amplitudes for the electromagnetic excitation of the baryon resonances and the elastic nucleon form factors. The goal is to describe data, if possible, but mainly to understand the mechanisms underlying the hadron dynamics, with particular attention to what is missing and should be introduced in the model in order to improve the agreement with data.

\section{The Model}

The hCQM, as other ones proposed in the literature [2, 3], is based on the idea of Constituent Quarks [4 (CQ). CQs are effective degrees of freedom, which describe the main quantum numbers of hadrons, but, at variance with QCD quarks, may acquire mass and even size.

It has been observed [5] that, according to Lattice QCD calculations [6], the quark interaction contains a long range spin-independent confinement and a short range spin dependent term. The latter can be attributed to the one-gluon 


\begin{tabular}{lccccc}
\hline$\left(56,0^{+}\right)$ & $P_{11}(938)$ & $P_{33}(1232)$ & & & \\
\hline$\left(56 *, 0^{+}\right)$ & $P_{11}(1440)$ & $P_{33}(1600)$ & & & \\
\hline$\left(70,1^{-}\right)$ & $D_{13}(1520)$ & $S_{11}(1535)$ & $S_{11}(1650)$ & $D_{13}(1700)$ & \\
& & $S_{31}(1620)$ & $D_{15}(1675)$ & $D_{33}(1700)$ & \\
\hline$\left(56,2^{+}\right)$ & $F_{15}(1680)$ & $P_{13}(1720)$ & $F_{35}(1910)$ & $P_{33}(1920)$ & $F_{37}(1950)$ \\
\hline$\left(70,0^{+}\right)$ & $P_{11}(1710)$ & $P_{31}(1910)$ & & & \\
\hline
\end{tabular}

Table 1: The SU(6) multiplets of the lower non strange baryon states

exchange interaction and treated as a perturbation. The dominance of the spin independent term allows to group the three-quark states into $\mathrm{SU}(6)$-multiplets. The baryon states are given by the product of three quark states, each being given by a six-component spinor. The three quark states can be decomposed into irreducible representations of $\mathrm{SU}(6)$ according to the scheme:

$$
6 \otimes 6 \otimes 6=20 \oplus 70 \oplus 70 \oplus 56
$$

where the representations have been labeled by their dimensions $d$. Introducing the total orbital angular momentum $L$ and the parity $\pi$, the $\mathrm{SU}(6)$ configurations can be labeled by $\left(d, L^{\pi}\right)$.In Table (1) we show how the observed non strange baryon states can be arranged into $\mathrm{SU}(6)$ multiplets.

The internal quark motion is described by the Jacobi coordinates

$$
\vec{\rho}=\frac{1}{\sqrt{2}}\left(\vec{r}_{1}-\vec{r}_{2}\right), \quad \vec{\lambda}=\frac{1}{\sqrt{6}}\left(\vec{r}_{1}+\vec{r}_{2}-2 \vec{r}_{3}\right),
$$

or equivalently, $\rho, \Omega_{\rho}, \lambda, \Omega_{\lambda}$. In order to describe the three-quark dynamics it is convenient to introduce the hyperspherical coordinates, which are obtained substituting the absolute values $\rho$ and $\lambda$ by

$$
x=\sqrt{\vec{\rho}^{2}+\vec{\lambda}^{2}}, \quad t=\operatorname{arctg}\left(\frac{\rho}{\lambda}\right),
$$

where $x$ is the hyperradius and $t$ the hyperangle. In this way the angularhyperangular part of the three quark wave functions is described by the hyperspherical harmonics (h.h.) [8] $Y_{[\gamma]}(\Omega)$, which are eigenstates of $L^{2}(\Omega)$, the Casimir operator of $O(6)$ :

$$
L^{2}(\Omega) Y_{[\gamma]}(\Omega)=-\gamma(\gamma+4) Y_{[\gamma]}(\Omega)
$$

where $\gamma=2 n+l_{\lambda}+l_{\rho}$, $\mathrm{n}$ being a non zero integer, $l_{\lambda}$ and $l_{\rho}$ the quark orbital angular momenta associated with the respective Jacobi variables and $\Omega$ denotes the angular-hyperangular variables.

The quark interaction can be expanded in a h.h. series, the first term depending on the hyperradius $x$ only:

$$
\Sigma_{i<j} V\left(r_{i j}\right)=V(x)+\ldots
$$


In the hypercentral constituent quark model (hCQM), the quark potential is assumed to depend on the hyperradius $x$ only, that is to be hypercentral. Therefore, $V=V(x)$ is in general a three-body potential, since the hyperradius $x$ depends on the coordinates of all the three quarks. In the three-quark wave function one can then factor out the hyperangular part $Y_{[\gamma]}(\Omega)$. The remaining hyperradial part of the wave function $\psi_{\gamma, \nu}(x)$ is determined by the hypercentral Schrödinger equation:

$$
\frac{d^{2}}{d x^{2}}+\frac{5}{x} \frac{d}{d x}-\frac{\gamma(\gamma+4)}{x^{2}} \psi_{\gamma, \nu}(x)=-2 m[E-V(x)] \psi_{\gamma, \nu}(x),
$$

where $v$ is a non negative integer number counting the number of nodes.

There are two hypercentral potentials which lead to analytical solutions. First, the h.o. potential, which has a two-body character, turns out to be exactly hypercentral, since

$$
\sum_{i<j} \frac{1}{2} k\left(\overrightarrow{r_{i}}-\overrightarrow{r_{j}}\right)^{2}=\frac{3}{2} k x^{2}=V_{\text {h.o. }}(x) .
$$

The second one is the 'hypercoulomb' potential

$$
V_{h y c}(x)=-\frac{\tau}{x} .
$$

This potential is not confining, however it is interesting [7 because it has an exact degeneracy between the first $0^{+}$excited state and the first $1^{-}$states [9, 10, 11, which can be respectively identified with the Roper resonance and the negative parity resonances. This degeneracy seems to be in agreement with phenomenology and is typical of an underlying $\mathrm{O}(7)$ symmetry [11. This feature cannot be reproduced in models with only two-body forces and/or harmonic oscillator bases since the excited $L=0$ state, having one more node, lies above the $L=1$ state 9 .

In the hCQM the quark potential is assumed in the form [1]

$$
V(x)=-\frac{\tau}{x}+\alpha x \quad,
$$

that means a coulomb-like term plus a linear confining term as suggested by lattice QCD calculations [12. In order to describe the splittings within the $S U(6)$-multiplets we introduce a hyperfine interaction $H_{h y p}$ of the standard form [2] and we treat it as a perturbation. The three quark hamiltonian is given then

$$
H=\frac{p_{\lambda}^{2}}{2 m}+\frac{p_{\rho}^{2}}{2 m}-\frac{\tau}{x}+\alpha x+H_{\text {hyp }}
$$

Having fixed the quark mass $m$ to $1 / 3$ of the nucleon mass, the remaining three free parameters $(\tau, \alpha$ and the strength of the hyperfine interaction) are fitted to the spectrum. The strength of the hyperfine interaction is determined by the $\Delta$ - Nucleon mass difference and the remaining parameters are given by $\tau=4.59$ and $\alpha=1.61 \mathrm{fm}^{-2}[1$. 
The confinement part of the interaction is not so effective in the low $x$ region, where the quark wave function is mainly concentrated and can therefore be treated perturbatively [13]; in this way, with a simplified form of the spin interaction, the model can be be formulated in an analytical approach [13. The $\mathrm{SU}(6)$ violation can be given also by an isospin dependent term [14, leading to a substantial improvement of the spectrum.

However in the following we shall use the interaction given by Eq. 10. Having fixed the parameters of the potential, the wave functions of the various resonances are completely determined and can be used for the prediction of various electromagnetic baryon properties.

\section{The helicity amplitudes}

The electromagnetic transition amplitudes, $A_{1 / 2}, A_{3 / 2}$ and $S_{1 / 2}$, are defined as the matrix elements of the quark electromagnetic interaction, $A_{\mu} J^{\mu}$, between the nucleon, $N$, and the resonance, $B$, states:

$$
\begin{aligned}
& A_{1 / 2}=\sqrt{\frac{2 \pi \alpha}{k}}\left\langle B, J^{\prime}, J_{z}^{\prime}=\frac{1}{2}\left|J_{+}\right| N, J=\frac{1}{2}, J_{z}=-\frac{1}{2}\right\rangle \\
& A_{3 / 2}=\sqrt{\frac{2 \pi \alpha}{k}}\left\langle B, J^{\prime}, J_{z}^{\prime}=\frac{3}{2}\left|J_{+}\right| N, J=\frac{1}{2}, J_{z}=\frac{1}{2}\right\rangle \\
& S_{1 / 2}=\sqrt{\frac{2 \pi \alpha}{k}}\left\langle B, J^{\prime}, J_{z}^{\prime}=\frac{1}{2}\left|J_{z}\right| N, J=\frac{1}{2}, J_{z}=-\frac{1}{2}\right\rangle
\end{aligned}
$$

$J_{\mu}$ is the electromagnetic current carried by quarks and will be used in its non relativistic form [15, 16, $k$ is the photon momentum in the Breit frame.

The results for the photocouplings, that is the transverse amplitudes with zero photon tetramomentum $\left(Q^{2}=0\right)$, the results of the hCQM compare favourably with other model [17]. The overall trend is reproduced, but all models suffer of a lack of strength; the similarities among the various models can be ascribed to a common underlying $\mathrm{SU}(6)$ structure.

The three-quark wave functions calculated with the hCQM of Eq. 10 can be used for the prediction of the $Q^{2}$ behavior of the helicity amplitudes [18]. The results for the transverse excitation to the negative parity resonances have been already published [18, while a systematic calculation of all excitations, both transverse and longitudinal will appear soon [19].

As an example, the hCQM results for the D13(1520) and the S11(1535) resonances [18, are given in Fig. 1 and 2, respectively. The agreement in the case of the S11 is remarkable, the more so since the hCQM curve has been published [18] well in advance with respect to the recent TJNAF data. In general the $Q^{2}$ behaviour is reproduced, except for discrepancies at small $Q^{2}$, especially in the $A_{3 / 2}^{p}$ amplitude of the transition to the $D_{13}(1520)$ state. The kinematical relativistic corrections at the level of boosting the nucleon and the resonances states to a common frame do not modify substantially the non-relativistic results 


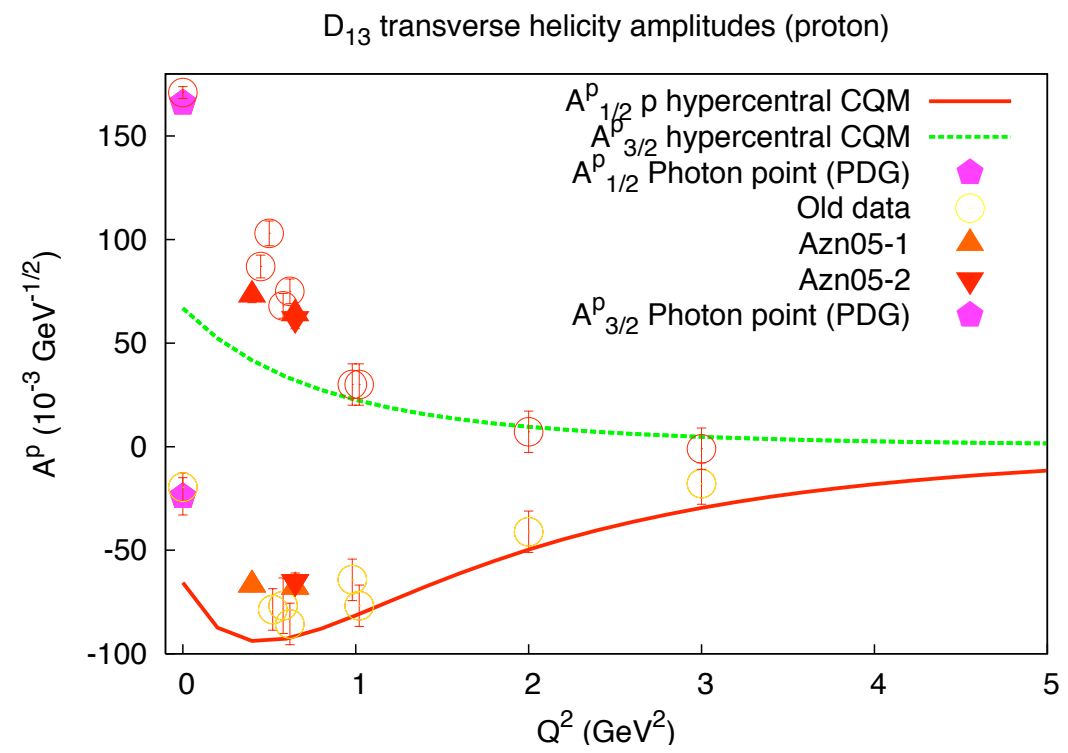

Figure 1: (Color online) The transverse helicity amplitudes for the D13(1520) resonance, calculated with the hCQM of Eq. (10). Data are from [20, 21, 22]. The photon point is also shown [23].

[27. Therefore these discrepancies can be ascribed to the lack of explicit quarkantiquark configurations $\left[18,28\right.$, which are expected to be important at low $Q^{2}$

The hCQM seems to provide realistic three-quark wave functions and the main reason is the presence of the hypercoulomb term. In fact, using the analytical version of the model [13, the wave functions in lower order coincide with the ones given by the hypercoulomb potential of Eq. (8), nevertheless the results for the helicity amplitudes are very similar to the ones predicted by the hCQM.

\section{The elastic nucleon form factors}

Recently at Jlab data [29] the ratio

$$
R_{p}=\mu_{p} \frac{G_{E}^{p}\left(Q^{2}\right)}{G_{M}^{p}\left(Q^{2}\right)}
$$

has been obtained directly from the polarization asymmetry measured in the elastic scattering of polarized electrons on polarized protons. This ratio deviates strongly from zero with increasing values of $Q^{2}$ and seems to tend to vanish at high $Q^{2}$. The problem arises of discrepancies with respect to data obtained with the Rosenbluth plot, however the Jlab data have triggered a renewed interest in 


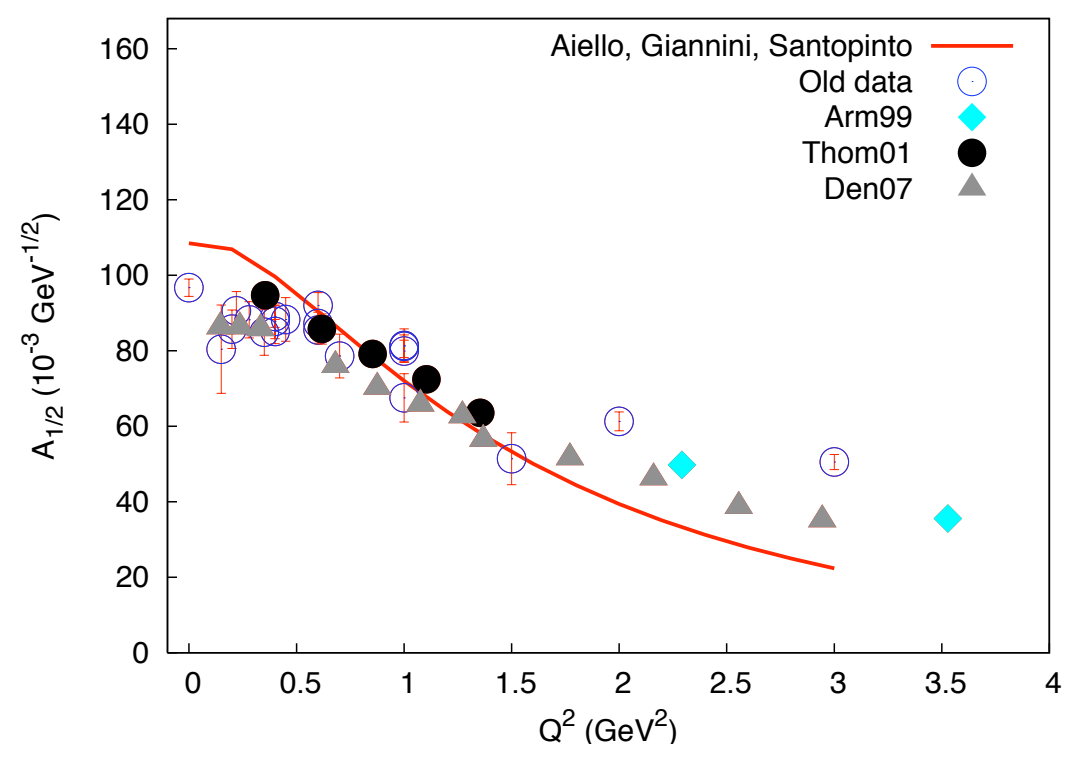

Figure 2: (Color online) The transverse helicity amplitude for the S11(1525) resonance, calculated with the hCQM of Eq. (10). Data are from [20, 24, 25, 26]. The photon point is also shown 23 .

the problem of describing the nucleon elastic form factors within quark models. In particular, the attention is focused on the possible zero of the electric form factor at high $Q^{2}$. It should be mentioned that extrapolating at high $Q^{2}$ the fit proposed many years ago [30, one gets a strong depletion of the ratio $R_{p}$ and a zero not far from $10 \mathrm{GeV}^{2}$. Calculations based on microscopic model for the nucleon structure provide a decreasing behavior for $R_{p}$, in some cases giving rise also to a zero 31 .

The hCQM provides nucleon wave functions which can be used for the calculation of the elastic form factor. However, since the calculated proton radius turns out to be about $0.5 \mathrm{fm}$, the resulting form factors are not good and one can expect that relativity is needed. In fact, we have shown that the depletion of $R_{p}$ is a relativistic effect [32] by simply boosting [33] the nucleon states to the Breit system one gets a deviation from 1 which reaches the value of about $15 \%$ at $Q^{2}=2 \mathrm{GeV}^{2}$.

For this reason, the hCQM has been fully relativized 34] using the Dirac Relativistic dynamics in the point form $(\mathrm{PF})$. The resulting predicted form factors are in good agreement, specially the magnetic ones, however, the ratio tends to a constant value of about 0.6 , with a behavior different from the observed one.

In order to understand what is missing, one can remind that CQs are effective degrees of freedom, which can acquire mass and even size. The latter statement 


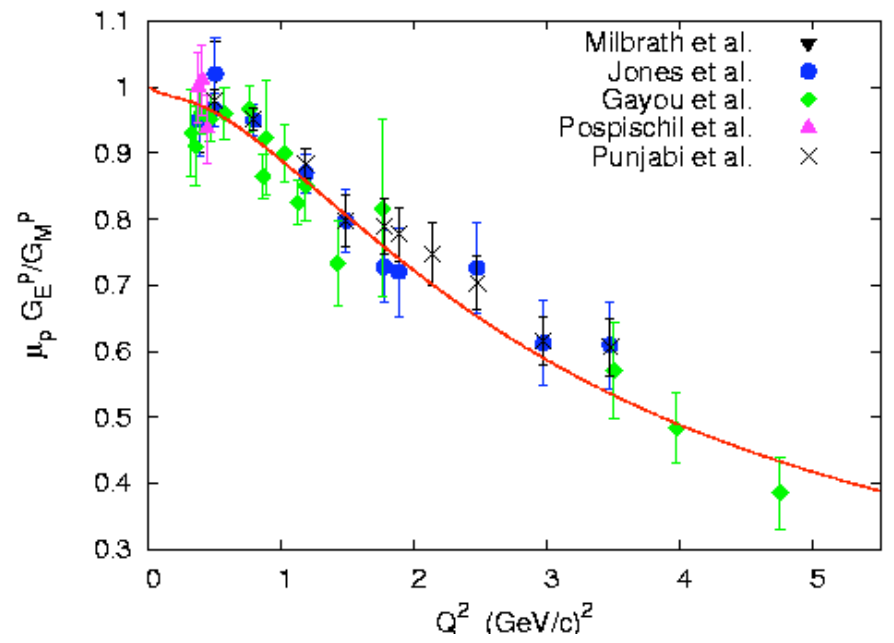

Figure 3: (Color online) The ratio $\mu_{p} G_{E}^{p} / G_{M}^{p}$ from polarization transfer compared with the relativistic hCQM calculation with constituent quark form factors (solid line).The experimental data are taken from 29. The Figure is taken from [34] (APS Copyright).

is supported by the recent analysis of the deep inelastic electron-proton scattering 35, in which there is evidence of quarks having a finite size. One can therefore think that CQ form factors must have a role also in the description of the nucleon from factors.

In Fig. 3 we report the relativistic hCQM result taking into account quark form factors. They have determined in order to reproduce at the same time the ratio $R_{p}$ and the proton magnetic, neutron electric and magnetic form factors up to $Q^{2}=5 \mathrm{GeV}^{2}$, with very nice results 34 .

The data seem to have a linearly decreasing behavior and the question is if they reach zero or not. A further information is given by very recent data [36], which extended the measure of $R_{p}$ up to $8.5 \mathrm{GeV}^{2}$. For this reason we have extended the calculation with the hCQM 34 up to $12 \mathrm{GeV}^{2}$ [37, without modifying any parameter. In order to study the high $Q^{2}$ behavior of $R_{p}$ one can alternatively consider the ratio

$$
F_{p}=Q^{2} \frac{F_{2}^{p}\left(Q^{2}\right)}{F_{1}^{p}\left(Q^{2}\right)}
$$

The zero of $R_{p}$ occurs when $F_{p}=4 M_{p}^{2}, M_{p}$ being the proton mass. In Fig. 4 we report the predicted high $Q^{2}$ behavior in comparison with the new data. While the first two new data are in agreement with the hCQM prediction, which does 


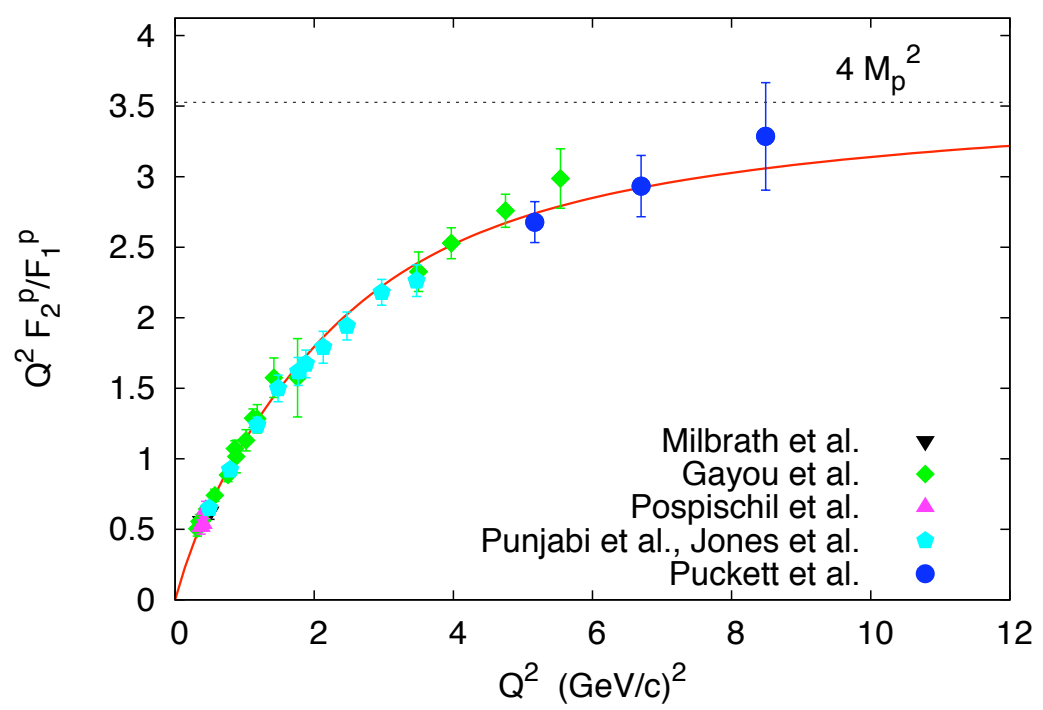

Figure 4: (Color online) The ratio $\mu_{p} G_{E}^{p} / G_{M}^{p}$ from polarization transfer compared with the relativistic hCQM calculation with constituent quark form factors (solid line).The experimental data are taken from [29, 36]. The Figure is taken from [37] (APS Copyright).

not tend towards a zero value of $R_{p}$, the last point is compatible with a dip in the electric form factor. The situation will be hopefully clarified by the future experiments to be performed at Jlab.

\section{Conclusion}

The hCQM seems to provide realistic quark wave functions even in its non relativistic formulation. Its predictions give rise to an overall agreement with the observed helicity amplitudes, specially in the medium $Q^{2}$ region, where the quark degrees of freedom are expected to dominate. At low $Q^{2}$ there is a lack of strength, which can be attributed to the missing quark-antiquark effects. The relativization of the model is certainly an important issue, although it appears to give relevant contributions in the case of the elastic form factor, while the helicity amplitudes are only slightly affected. The missing dynamical mechanism is given by the quark-antiquark creation, pointing towards the unquenching of the CQMs. Important progress in this direction is provided by recent work, in particular for the baryons by [38, 39], while for the mesons by [40. Such unquenched CQs will allow a consistent treatment of the baryon spectrum, the elastic nucleon properties and the electroproduction of mesons. 


\section{References}

[1] M. Ferraris, M.M. Giannini, M. Pizzo, E. Santopinto and L. Tiator, Phys. Lett. B364, 231 (1995).

[2] N. Isgur and G. Karl, Phys. Rev. D18, 4187 (1978); D19, 2653 (1979); D20, 1191 (1979).

[3] S. Capstick and N. Isgur, Phys. Rev. D 34,2809 (1986); R. Bijker, F. Iachello and A. Leviatan, Ann. Phys. (N.Y.) 236, 69 ( 1994); L. Ya. Glozman and D.O. Riska, Phys. Rep. C268, 263 (1996); L. Ya. Glozman, Z. Papp, W. Plessas, K. Varga, R. F. Wagenbrunn, Phys. Rev. C57, 3406 (1998); L. Ya. Glozman, W. Plessas, K. Varga, R. F. Wagenbrunn, Phys. Rev. D58, 094030 (1998); U. Löring, K. Kretzschmar, B. Ch. Metsch, H. R. Petry, Eur. Phys. J. A10, 309 (2001); U. Löring, B.Ch. Metsch, H. R. Petry, Eur. Phys. J. A10, 395 (2001); 447 (2001));E. Santopinto, Phys. Rev. C 72 (2005) 022201; J. Ferretti, A. Vassallo and E. Santopinto, Phys. Rev. C 83 (2011) 065204; M. De Sanctis, J. Ferretti, E. Santopinto and A. Vassallo, Phys. Rev. C 84 (2011) 055201.

[4] G. Morpurgo, 1965 Physics 2, 95 (1965).

[5] A. De Rújula, H. Georgi and S.L. Glashow, Phys. Rev. D12, 147 (1975).

[6] K. Wilson, Phys. Rev. D 10, 2445 (1974); J. Kogut, L. Susskind, Phys. Rev. D 9, 3501 (1974).

[7] R. Bijker, F. Iachello and E. Santopinto, J. Phys. A: Math. Gen. 31, 9041 (1998)

[8] J. Ballot and M. Fabre de la Ripelle, Ann. of Phys. (N.Y.) 127, 62 (1980).

[9] J.-M. Richard, Phys. Rep. C 212, 1 (1992).

[10] M. Fabre de la Ripelle and J. Navarro, Ann. Phys. (N.Y.) 123, 185 (1979).

[11] E. Santopinto, M.M. Giannini and F. Iachello, in "Symmetries in Science VII", ed.B. Gruber, Plenum Press, New York, 445 (1995); F. Iachello, in "Symmetries in Science VII", ed. B. Gruber, Plenum Press, New York, 213 (1995).

[12] G. Bali et al., Phys. Rev. D 51,5165 (1995); G. Bali, Phys. Rept. 343, 1 (2001); C. Alexandrou, P. de Forcrand and O. Jahn, Nucl. Phys. Proc. Suppl. 119,667 (2003); H. Suganuma, T. T. Takahashi, F. Okiharu and H. Ichie, Nucl. Phys. Proc. Suppl. 141, 92 (2005).

[13] E. Santopinto, F. Iachello and M. M. Giannini, Eur. Phys. J. A 1, 307 (1998); E. Santopinto, F. Iachello and M. M. Giannini, Nucl. Phys. A 623, 100c (1997).

[14] M.M. Giannini, E. Santopinto, A. Vassallo, Eur. Phys. J. A12, 447 (2001). 
[15] L. A. Copley, G. Karl and E. Obryk, Phys. Lett. 29, 117 (1969).

[16] R. Koniuk and N. Isgur, Phys. Rev. D21, 1868 (1980).

[17] M. Aiello, M. Ferraris, M.M. Giannini, M. Pizzo and E. Santopinto, Phys. Lett. B387, 215 (1996).

[18] M. Aiello, M. M. Giannini, E. Santopinto, J. Phys. G: Nucl. Part. Phys. 24, 753 (1998)

[19] E. Santopinto, M.M. Giannini, A systematic study of longitudinal and transverse helicity amplitudes in the hypercentral Constituent Model, to be publihed.

[20] V. Burkert, private communication.

[21] I.G. Aznauryan et al., Phys. Rev. C 71, 015201 (2005).

[22] I. G. Aznauryan, V. D. Burkert, G. V. Fedotov, B. S. Ishkhanov, V. I. Mokeev, Phys. Rev. C 72045201 (2005).

[23] K. Nakamura et al. (Particle Data Group), J. Phys. G: Nucl. Part. Phys. 37, 075021 (2010).

[24] C.S. Armstrong et al., Phys. Rev. D 60, 052004 (1999).

[25] R. Thompson et al. (Clas Collaboration), Phys. Rev. Lett. 86, 1702 (2001).

[26] H. Denizli et al. (Clas collaboration), Phys. Rev. C 76, 015204 (2007).

[27] M. De Sanctis, E. Santopinto, M.M. Giannini, Eur. Phys. J. A2, 403 (1998).

[28] E. Santopinto, Ph. D. Thesis (Genova 1995).

[29] B. D. Milbrath et al. [Bates FPP collaboration], Phys. Rev. Lett. 80 (1998) 452 [Erratum-ibid. 82 (1999) 2221]; M. K. Jones et al. [Jefferson Lab Hall A Collaboration], Phys. Rev. Lett. 84, 1398 (2000); O. Gayou et al. , Phys. Rev. C 64, 038202 (2001); O. Gayou et al. [Jefferson Lab Hall A Collaboration], Phys. Rev. Lett. 88, 092301 (2002); T. Pospischil et al. [A1 Collaboration], Eur. Phys. J. A 12 (2001) 125; V. Punjabi et al., Phys. Rev. C 71, $055202(2005)$.

[30] F. Iachello, A. D. Jackson and A. Landé, Phys. Lett. B 43, 191 (1973).

[31] G. Holzwarth, Z. Phys. A 356, 339 (1996); G. Holzwarth, Nucl. Phys. A 666, :24 (2000); M. R. Frank, B. K. Jennings and G. A. Miller, Phys. Rev. C 54, 920 (1996); G.A. Miller, Phys. Rev. C 66, 032201(R) (2002); F. Cardarelli and S. Simula, Phys. Rev. C 62, 065201 (2000); J.P.B.C. deMelo, T. Frederico, E. Pace, G. Salmé, Phys. Lett. bf B 581, 75 (2004); Phys. Rev. D 73074013 (2006); J.P.B.C. deMelo, T. Frederico, E. Pace, S. Pisano, G. Salmé, Phys. Lett. bf B 671, 153 (2009); R. F. Wagenbrunn, S. Boffi, 
W. Klink, W. Plessas and M. Radici, Phys. Lett. B 511, 33 (2001); S. Boffi, L. Y. Glozman, W. Klink, W. Plessas, M. Radici and R. F. Wagenbrunn, Eur. Phys. J. A 14, 17 (2002).

[32] M. De Sanctis, M. M. Giannini, L. Repetto and E. Santopinto, Phys. Rev. C 62, 025208 (2000).

[33] M. De Sanctis, E. Santopinto, M.M. Giannini, Eur. Phys. J. A1, 187 (1998).

[34] M. De Sanctis, M. M. Giannini, E. Santopinto, A. Vassallo, Phys. Rev. C 76, 062201(R) (2007); M. De Sanctis, M. M. Giannini, E. Santopinto and A. Vassallo, Eur. Phys. J. A 19 (2004) 81.

[35] R. Petronzio, S. Simula, G. Ricco, Phys. Rev. D67, 094004 (2003), Erratum-ibid. D68, 099901 (2003).

[36] A. J. R. Puckett et al., Phys. Rev. Lett. 104, 242301 (2010).

[37] E. Santopinto, A. Vassallo, M. M. Giannini, M. De Sanctis, Phys. Rev. C 82, $065204(2010))$.

[38] E. Santopinto, R. Bijker, Few-Body Syst. 4495 (2008); R. Bijker, E. Santopinto, Phys. Rev. C80, 065210 (2009); E. Santopinto and G. Galata, Phys. Rev. C 75 (2007) 045206; S. Capstick et al., Eur. Phys. J. A 35 (2008) 253;E. Santopinto, R. Bijker, Phys. Rev. C82 062202 (2010); E. Santopinto, R. Bijker, J. Ferretti, Few-Body Syst. 50199 (2011); R. Bijker, J. Ferretti, E. Santopinto, Phys. Rev. C85 035204 (2012).

[39] E. Santopinto, Contrinution to this Workshop.

[40] J. Ferretti, G. Galatà , E. Santopinto, A. Vassallo,Phys. Rev. C85 035204 (2012). 